\title{
Active control for medium-frequency transformers flux-balancing in a novel three-phase topology for cascaded conversion structures
}

\author{
David Lumbreras $^{1}$, Ernesto L. Barrios ${ }^{1}$, Manuel Navarrete ${ }^{2}$, Julian Balda ${ }^{2}$, Roberto Gonzalez ${ }^{1,2}$, Pablo Sanchis ${ }^{1}$ \\ ${ }^{I}$ Department of Electrical, Electronic and Communication Engineering \\ Public University of Navarre (UPNA) \\ Insitute of Smart Cities \\ Pamplona, Spain \\ ${ }^{2}$ Department of Photovoltaic Solar Energy \\ Ingeteam Power Technology S.A. \\ Sarriguren, Spain
}

\begin{abstract}
Efficiency and power density are important Therefore, a way to avoid the saturation of MF and HF parameters in the design of power electronics converters. In transformers without compromising the power density of the many applications, low-frequency transformers are being converter is one of the main issues to be addressed in these substituted for medium-frequency and high-frequency new topologies.

transformers in order to reduce the volume and therefore the cost of the transformer. However, preventing their saturation is a The main purpose of this paper is to study the MF complex task. This paper studies the medium-frequency transformers's flux evolution in a three-phase topology for transformers' flux balancing in a novel three-phase topology for cascaded conversion structures, and to propose an active cascaded conversion structures. Based on the modulation control technique to prevent its saturation. The basic operation technique of the converter, a method to directly measure the of the topology is explained in Section II (it is described in magnetizing current of the medium-frequency transformers is detail in [6]). In Section III, the technique to measure the proposed in this paper. A control loop to regulate the de value of magnetizing current and the control loop to prevent the the magnetizing current is also designed and developed. saturation of the MF transformers are presented and Simulation results validate the correct operation of the control explained. Simulation results that validate the proposed loop, which prevents the transformer saturation. technique are shown in section IV.
\end{abstract}

Keywords-high-frequency transformer, medium-frequency transformer, saturation, magnetizing current, photovoltaic system.

\section{INTRODUCTION}

Due to the massive use of renewable energies [1], efficiency and power density are becoming even more important in the power converter design process [2]. When galvanic isolation is needed, the low-frequency (LF) transformer is one of the biggest and heaviest components of the energy conversion stage [3]. However, as it cannot be eliminated due to isolation requirements, new conversion topologies are trying to substitute LF transformers for medium-frequency (MF) or high-frequency (HF) transformers, in order to reduce the volume and weight of the power conversion structure [4].

Regarding photovoltaic systems (PV), cascaded mediumvoltage topologies are being investigated [4]. They consist of multiple converters whose outputs are connected in series, in which the traditional line-frequency transformers are replaced by MF or HF transformers. However, saturation of MF and HF transformers can easily happen even if the volt-seconds imbalance is very small [5]. If saturation occurs, a magnetizing current with high peak values appears, resulting in an overcurrent that may break the semiconductors. Furthermore, saturation increases the losses both in the core and the windings, which can lead to the transformer destruction. The core can be oversized to avoid the transformer saturation, but it reduces the power-density of the converter and increases its cost. Other passive methods, such as including series capacitors, are not advisable in high power or high power-density converters for the same reason.

\section{THREE-PHASE TOPOLOGY FOR CASCADED CONVERSION} STRUCTURES

Fig. 1 summarizes the PV plant configuration of a cascaded conversion system in a large-scale photovoltaic plant. It has $N_{s}$ three-phase conversion stages, whose ac outputs are connected in series. The topology of each one of the $N_{s}$ conversion stages is shown in Fig. 2. It is formed by an $\mathrm{H}$-bridge as a dc/ac converter, 3 single-phase MF frequency transformers with turns ratio of $N_{1} / N_{2}$, and three ac/ac converters (one per phase), as shown in Fig. 2.

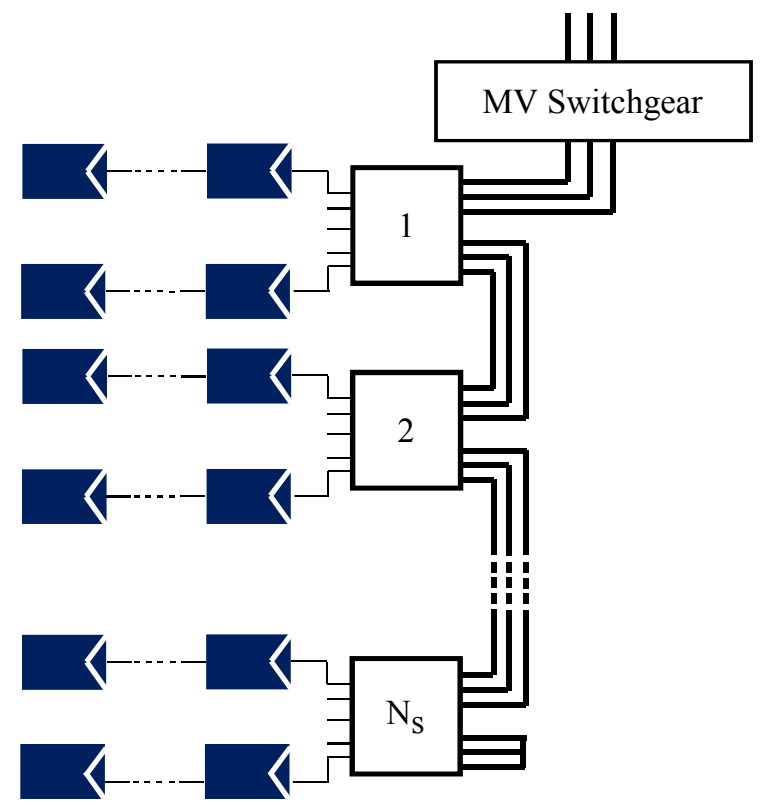

This work was supported by the Spanish State Research Agency (AEI) under grants PID2019-110956RB-I00 /AEI/ 10.13039/501100011033 and Fig. 1. PV plant configuration of the proposed cascaded conversion structure DPI-2016-80641-R, and by the Public University of Navarre through a doctoral scolarship

(C) 2020 IEEE. Personal use of this material is permitted. Permission from IEEE must be obtained for all other uses, in any current or future media, including reprinting/republishing this material for advertising or promotional purposes, creating new collective works, for resale or redistribution to servers or lists, or reuse of any copyrighted component of this work in other work. 


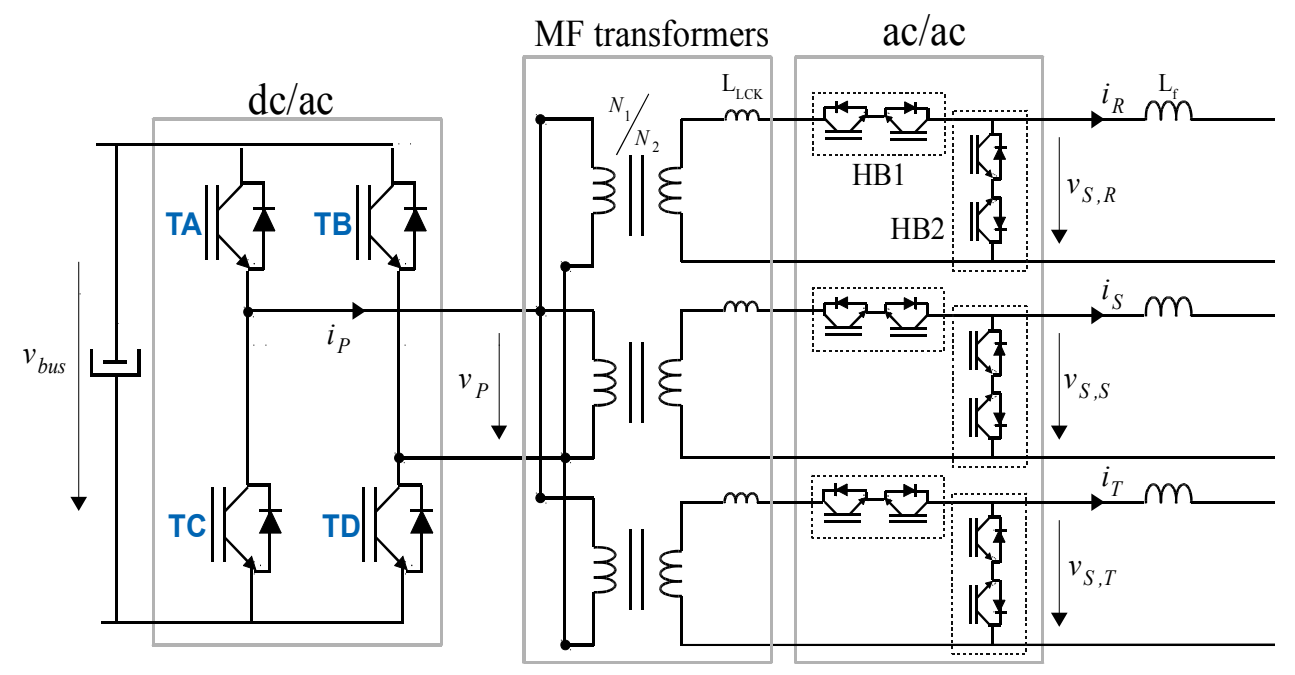

Fig. 2. Three-phase topology configuration of each conversion stage

The basic operation of the converter is explained in Fig. 3. The topology and its modulation technique are detailed in [6]. The three phases are activated sequentially and one at a time, which means that in a switching period, $t_{S W}$, first the phase $\mathrm{R}$ is fed with power, then phase $\mathrm{S}$ and finally phase $\mathrm{T}$. When a phase is activated, the corresponding ac/ac converter connects the phase's output to the transformer $(\mathrm{HB} 1=\mathrm{ON}$ and $\mathrm{HB} 2=\mathrm{OFF}$ ) and the H-bridge applies the required voltage. When a phase is not enabled, it only permits the current flow to the rest of the conversion stages $(\mathrm{HB} 1=\mathrm{OFF}$ and $\mathrm{HB} 2=\mathrm{ON})$. This way, power is transferred sequentially from the input to the three outputs of the converter.

Like in a traditional converter, the time during which a phase is enabled depends on the modulation index (i.e. the comparison between a reference voltage and a carrier wave). With the proposed topology, as seen in Fig. 3, a positive, negative, or zero voltage can be obtained in each phase.

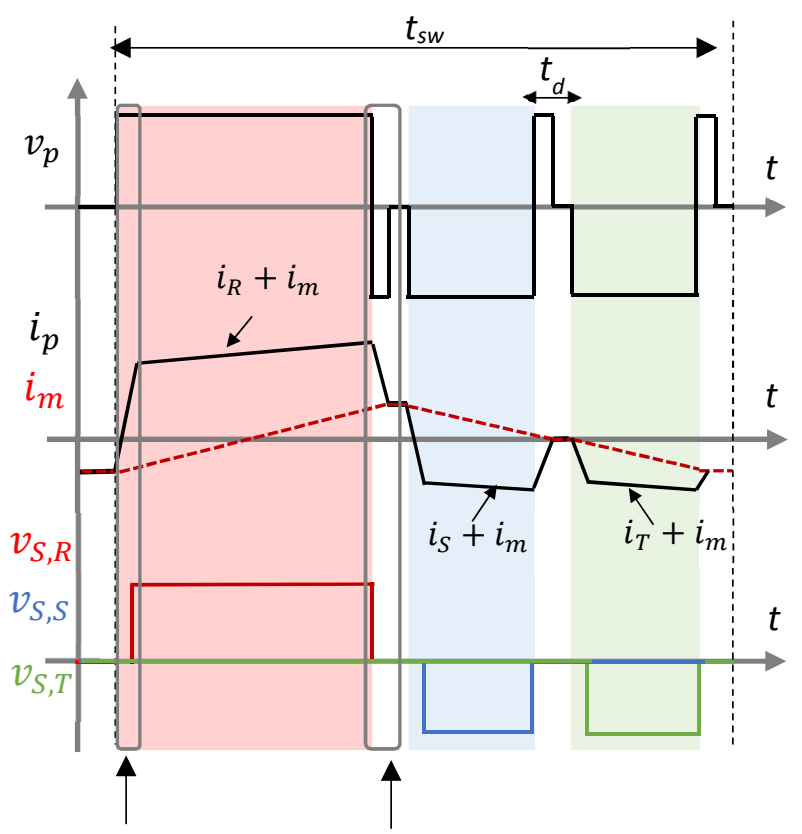

Phase R turn-on Phase R turn-off

Fig. 3. Basic operation of the proposed topology
Regarding the magnetization of the transformer, in a switching period, the voltage applied to the transformer, $v_{p}$, corresponds to the sequential control of the three phases. Ideally, in a switching period, the addition of the control voltages of the three phases is equal to zero. Therefore, $v_{p}$ has an average value of zero, and the magnetization of the transformer is done at switching frequency.

One of the main characteristics of the proposed threephase topology is its modulation technique, since the leakage inductance of the transformer, $L_{L C K}$, is series-connected with the output filter inductance, $L_{f}$. In this way, specific turn-on and turn-off transitions have been developed depending on the sign of the voltage that the H-bridge applies when the phase is active, and the sign of the grid current of the corresponding phase [6]. However, all the possible transitions have in common some important characteristics that affect the transformer saturation.

Firstly, as shown in Fig. 3, a dead time, $t_{d}$, always appears between the activation of a phase and the next one. The three dead times of a switching period slightly reduce the effective modulation index (i.e. the effective time that can be used to transfer power). However, high effective modulation indexes can be achieved, as will be shown in section IV. During the dead time, a voltage of opposite sign to that of the grid current is applied to the transformer. This way, the current in $L_{L C K}$ is extinguished and the phase is correctly deactivated. As a consequence, once the current is extinguished in $L_{L C K}$ and before the next phase is controlled, the current in the H-bridge, $i_{p}$, is constant and equal to the magnetizing current, $i_{m}$. This is used to measure the magnetizing current at the end of each dead time, as is further explained in section III. However, the voltage that is applied during the 3 dead times to extinguish the current in $L_{L C K}$, results in an effective voltage applied to the transformer with an average value different to zero. The sign of this effective voltage depends on the signs of the grid currents. For example, if $i_{R}$ is positive, and $i_{S}$ and $i_{T}$ are negative, a positive effective voltage is applied to the transformer, as shown in Fig. 3. Studying the evolution of the grid current's signs during a grid-frequency period, it can be determined that this characteristic of the modulation technique causes that a small-amplitude voltage component at $150 \mathrm{~Hz}$ is applied to the transformer. 
Another characteristic of the modulation technique [6] is that during the turn-on transitions, a voltage is applied to the leakage inductance of the transformer, $L_{L C K}$, so that the grid current starts flowing through $L_{L C K}$. However, as depicted in Fig. 3, only when all the grid current is passing through $L_{L C K}$ (i.e. $i_{R}$ is passing through $L_{L C K}$ ), a voltage appears at the output of the ac/ac converter. This fact generates an error in the output voltage, which depends on the sign and the value of the grid current, and whose effect is similar to the one of the dead times in a conventional inverter [7]. Consequently, the magnetizing current has small-amplitude low-frequency components, being the most important one the component at $50 \mathrm{~Hz}$.

Thus, on the one hand, the modulation technique allows to measure the magnetizing current three times per switching period, but on the other hand, it affects the transformer saturation, introducing low-frequency voltage components.

\section{PROPOSED CONTROL TECHNIQUE FOR PREVENTING THE MF TRANSFORMER SATURATION}

Transformers can saturate even if the difference between the positive and negative volts-seconds applied to the transformer is small [5]. If a transformer saturates, a magnetizing current with high peak values appears, resulting in an overcurrent that may break the semiconductors. This higher current also increases the transformer losses and might lead to its destruction. The core can be oversized to avoid saturation, but it reduces the power-density of the converter and increases its cost. A capacitor in series with the transformer is also not advisable in high power or high powerdensity converters due to the large capacitance required unless a resonant topology is used. In [5], different techniques to detect the transformer saturation are studied. For the proposed topology, if a high power-density converter is desired, saturation detection techniques with a parallel flux path [8], [9] cannot be considered, as they do not detect the saturation of the core until it has been reached. The integration of the voltage applied to the transformer [10] is also discarded for the proposed topology because, as the applied voltage has to be filtered, this technique is not fast enough to prevent the MF transformers saturation. A continuous and instantaneous flux measurement with an active control is proposed in this paper, which is much easier and simple than "The Magnetic Ear" [5], as it does not need additional magnetic paths and it reduces the complexity of the additional circuits needed to detect voltseconds imbalances.
Fig. 4 summarizes the proposed technique to measure the magnetizing current, where the three MF transformers have been substituted by their equivalent magnetic inductance, $L_{m} / 3$, and series resistance of the windings, $R_{t r} / 3$. In order to measure the current with high bandwidth, a shunt resistor, $R_{\text {shunt }}$, is placed between the $\mathrm{H}$-bridge and the MF transformers. This high bandwidth allows a small measuring time, which reduces the required dead time between phases, and therefore increases the effective modulation index. As explained in section II, thanks to the modulation of the converter, at the end of a turn-off transition, the current that is passing through $R_{\text {shunt }}$ is equal to the magnetizing current, $i_{m}$. This way, the voltage across the shunt resistor, is at the end of the dead time proportional to $i_{m}$.

The voltage across the shunt resistor has to be isolated before it is read by an analog-to-digital converter, ADC. For this purpose, the Si8920 isolated amplifier is used. It is an isolated amplifier specially designed for shunt resistors, which has a bandwidth of $750 \mathrm{kHz}$, one of the highest in the market. Again, to obtain a high effective modulation index it is very important to select an isolated amplifier with very high bandwidth.

The isolated voltage then goes to the input of a sample and hold, S\&H, which is activated at the end of each dead time as shown in Fig. 4. The acquisition time of the $\mathrm{S} \& \mathrm{H}$ is also very important to obtain a high effective modulation index. The AD781JNZ is selected for this application, as it has an acquisition time lower than $1 \mu \mathrm{s}$. In addition, the signal that enables the S\&H has to be correctly coordinated with the modulation of the converter so that the voltage is held only when the current in the H-bridge is equal to the magnetizing current and not before. This is done with an FPGA, which also controls the coordination between the semiconductors of the $\mathrm{H}$-bridge and the ac/ac converters.

The held signal of the S\&H is finally read by an analogto-digital converter, $\mathrm{ADC}$. The reason why a $\mathrm{S} \& \mathrm{H}$ is used is that the ADC might not be fast enough to quickly read and store the voltage value before the next phase starts its turn-on transiton. This way, first, the S\&H is enabled to quickly hold the voltage that corresponds to the magnetizing current, and then the ADC reads the held signal. This ensures that a voltage proportional to the magnetizing current is always measured in the ADC, even though the next phase might have started with its turn-on transition and $i_{p}$ is not equal to $i_{m}$. If a fast ADC is used, the $\mathrm{S} \& \mathrm{H}$ can be removed.
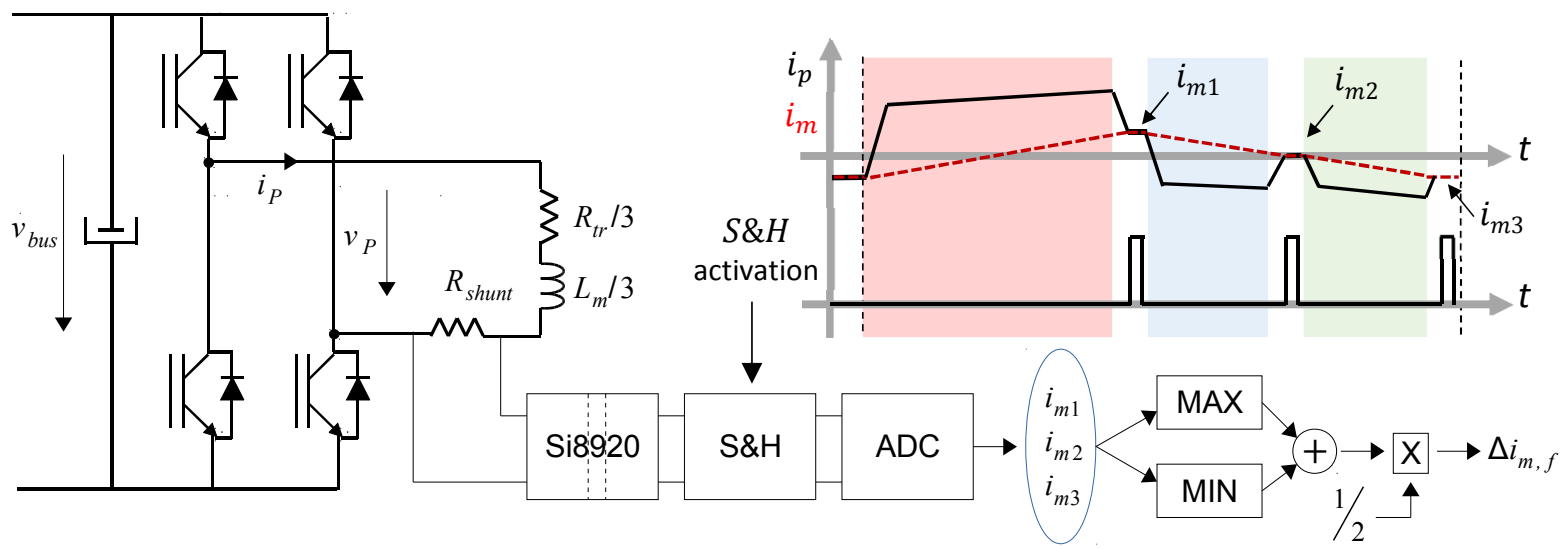

Fig. 4. Proposed technique to obtain the dc value of the magnetizing current 


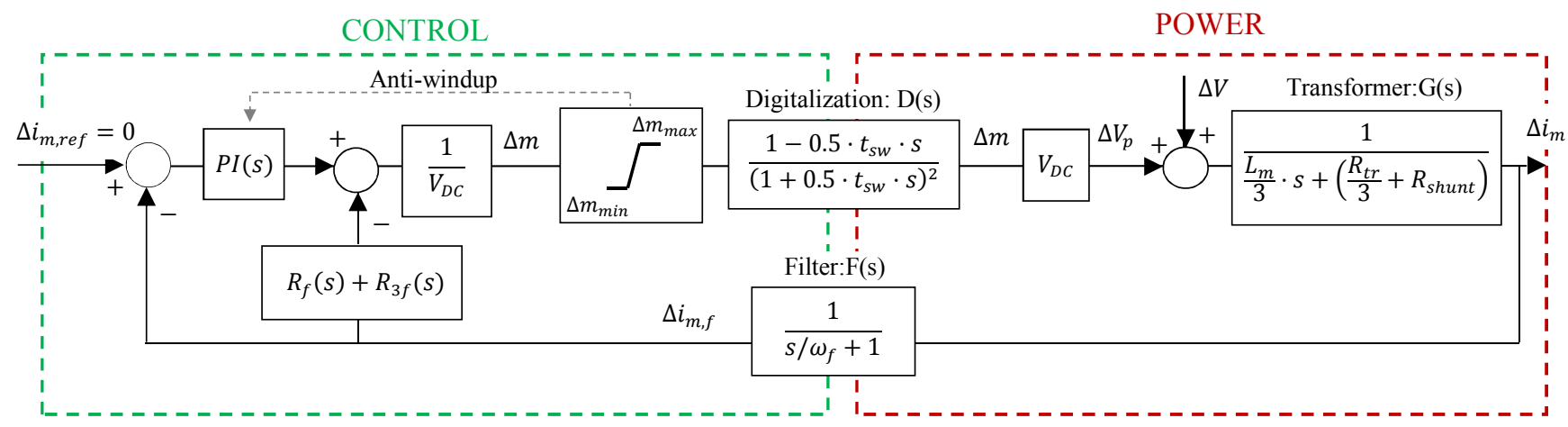

Fig. 5. Control loop to regulate the dc value of the magnetizing current

Thanks to the modulation technique of the converter, in a switching period, 3 values of the magnetizing current are obtained. If the highest and the lowest values are added and then divided by 2 , the dc value of the magnetizing current in that switching period is obtained, $\Delta i_{m, f}$. If it is equal to zero (i.e. highest and lowest measurements are equal but with opposite signs), the magnetizing current does not have a dc component. Otherwise, the magnetizing current has a dc value, indicating that an average voltage different to zero has been applied to the transformers during the switching period.

In order to avoid the transformers saturation, the voltseconds imbalances that are applied to the transformer have to be corrected. A control loop to regulate the dc value of the magnetizing current, $\Delta i_{m}$, is presented in Fig. 5. If a dc value in the magnetizing current is detected (i.e. $\Delta i_{m_{f}}$ is different to zero), it means that a voltage with an average value different to zero has been applied to the transformer. The PI controller then calculates the necessary modulation index variation, $\Delta m$, to compensate the flux imbalance. In particular, $\Delta m$ is divided into 3 and added to the reference voltages that are obtained in the current control loops. This way, the final reference voltages will cause that in the next switching period, a smallamplitude dc voltage is applied to the transformer, $\Delta V_{p}$, which will try to compensate the volt-seconds imbalance

The modulation index variation, $\Delta m$, is limited to a minimum and a maximum value, because otherwise the time for transferring power in a switching period, $t_{e f f}$, can highly be reduced if a big flux imbalance is suddenly detected. The limits of the modulation index variation are determined by the amount of time that can be used for the volts-seconds correction, and have to be chosen according to the application.

In the control loop of Fig. 5, the delays due to computation and the zero-order-hold are grouped in the transfer function $D(s)$ and modelled by a first order Padé approximation [11].

The current filter, $F(s)$, represents the filtering between the voltage across the shunt resistor and voltage that is read in the ADC. It can be modelled as a first-order low pass filter whose cutoff frequency, $\omega_{f}$, is equal to the bandwidth of the isolated amplifier.

The relationship between the voltage applied to the transformers, and the magnetizing current is modelled by the transfer function $G(s)$, which depends on the magnetic inductance of the transformers, $L_{m}$, the resistance of its windings, $R_{t r}$, and the shunt resistor used to measure the current, $R_{\text {shunt }}$, as it is placed in series with the transformers.
The control loop also includes two resonant terms to cancel the most important low-frequency voltage components that are applied to the transformer due to the modulation technique. As explained in section II, these components are at 50 and $150 \mathrm{~Hz}$. Thus, a resonant term for cancelling each component is included in the control loop, named respectively as $R_{f}(s)$ and $R_{3 f}(s)$ in Fig. 5. The low-frequency voltage components are represented in Fig. 5 by $\Delta V$. The design of the control loop and its validation are carried out in the following section.

\section{CONTROL TECHNIQUE VALIDATION}

A small-scale prototype of a converter with the proposed three-phase topology is studied. Its characteristics are detailed in Table I.

The time needed to extinguish the current in $L_{L C K}, t_{L C K}$, can be calculated as:

$$
t_{L C K}=L_{L C K} \cdot \frac{i_{\text {max }}}{v_{\text {bus } \text { min }} \cdot N_{2} / N_{1}}
$$

where $i_{\max }$ is the maximum grid current that has to be extinguished in $L_{L C K}$. In the prototype, $10 \mu \mathrm{s}$ are enough to guarantee that the current in $L_{L C K}$ is extinguished at any operating point of the converter.

A final value of $t_{d}=15 \mu \mathrm{s}$ is chosen for the turn-off transition. This way it is ensured that the $\mathrm{S} \& \mathrm{H}$ is activated only when $i_{m}=i_{p}$ and not before, and that the voltage is correctly held with the S\&H before the next turn-on transition starts.

TABLE I. PARAMETERS OF THE SMALL-SCALE PROTOTYPE

\begin{tabular}{c|c}
\hline \hline Rated power & $300 \mathrm{VA}$ \\
\hline$t_{s w}$ & $500 \mu \mathrm{s}$ \\
\hline$N_{1} / N_{2}$ & $1 / 2$ \\
\hline$v_{\text {bus, } \text { max }}$ & $60 \mathrm{~V}$ \\
\hline$v_{\text {bus, } \text { min }}$ & $45 \mathrm{~V}$ \\
\hline$L_{m}$ & $7.5 \mathrm{mH}$ \\
\hline$R_{t r}$ & $0.15 \mathrm{~m} \Omega$ \\
\hline$R_{\text {shunt }}$ & $6 \mathrm{~m} \Omega$ \\
\hline$L_{L C K}$ & $140 \mu \mathrm{H}$ \\
\hline$\omega_{f}$ & $750 \mathrm{kHz}$ \\
\hline Grid line voltage & $40 \mathrm{Vrms}$ \\
\hline \hline
\end{tabular}


Besides, a maximum value of $30 \mu \mathrm{s}$ is allowed for the volts-seconds correction, which corresponds to a maximum modulation voltage variation of $\Delta m_{\min }=-0.06$ and $\Delta m_{\max }=0.06$. Consequently, $t_{\text {eff }}$ is equal to $425 \mu \mathrm{s}$, obtaining a final effective modulation index of 0.85 .

With the parameters from Table I, the PI controller is firstly designed without taking into account the resonant terms, as they will only alter the frequencies close to their resonant frequencies. The open-loop transfer function between the dc value of the magnetizing current, $\Delta i_{m}$, and the reference, $\Delta i_{m, r e f}$, without considering the resonant terms, $L_{P I}(s)$, is:

$$
L_{P I}(s)=P I(s) \cdot D(s) \cdot G(s) \cdot F(s)
$$

The PI controller is designed for a phase margin of $65^{\circ}$ and a crossover frequency of $100 \mathrm{~Hz}$. The resulting Bode diagram of the open loop in (2) is shown in Fig. 6.

The transfer functions of the resonant terms at $50 \mathrm{~Hz}$, $R_{f}(s)$, and at $150 \mathrm{~Hz}, R_{3 f}(S)$, can be expressed as:

$$
\begin{gathered}
R_{f}(s)=K_{r_{f}} \cdot \frac{s \cdot \cos \theta_{f}-\omega_{0_{f}} \cdot \sin \theta_{f}}{s^{2}+2 \cdot \omega_{c_{f}} \cdot \omega_{0_{f}} \cdot s+\omega_{0_{f}}^{2}}, \\
R_{3 f}(s)=K_{r_{3 f} \cdot \frac{s \cdot \cos \theta_{3 f}-\omega_{0_{3 f}} \cdot \sin \theta_{3 f}}{s^{2}+2 \cdot \omega_{c_{3 f}} \cdot \omega_{0_{3 f}} \cdot s+\omega_{0_{3 f}}^{2}},},
\end{gathered}
$$

where $K_{r f}$ and $K_{r 3 f}$ are the gains of the resonant terms, $\omega_{0 f}$ and $\omega_{03 f}$ are its resonant frequencies (i.e. 50 and $150 \mathrm{~Hz}$ ), $\omega_{c f}$ and $\omega_{c 3 f}$ is related to its damping (chosen as $0.2 \%$ ), and $\theta_{f}$ and $\theta_{3 f}$ are the angles that are used for the delay compensation of the resonant terms in order to make the control loop stable [12].

The main purpose of these resonant terms is to cancel the small-amplitude low-frequency components that are introduced due to the modulation of the converter. Thus, for its design, the closed-loop transfer function between $\Delta V$ and $\Delta i_{m, r e f}, C L_{R E S}(S)$, is studied. It can be expressed as:

$$
C L_{R E S}(s)=\frac{G(s)}{1+G(s) \cdot D(s) \cdot\left(P I(s)+R_{f}(s)+R_{3 f}(s)\right) \cdot F(s)}
$$

The gain of the resonant terms must be high enough to ensure the cancelation of the harmonic components of $\Delta V$. Thus, $K_{r f}=100$ and $K_{r 3 f}=150$. The resulting Bode diagram of
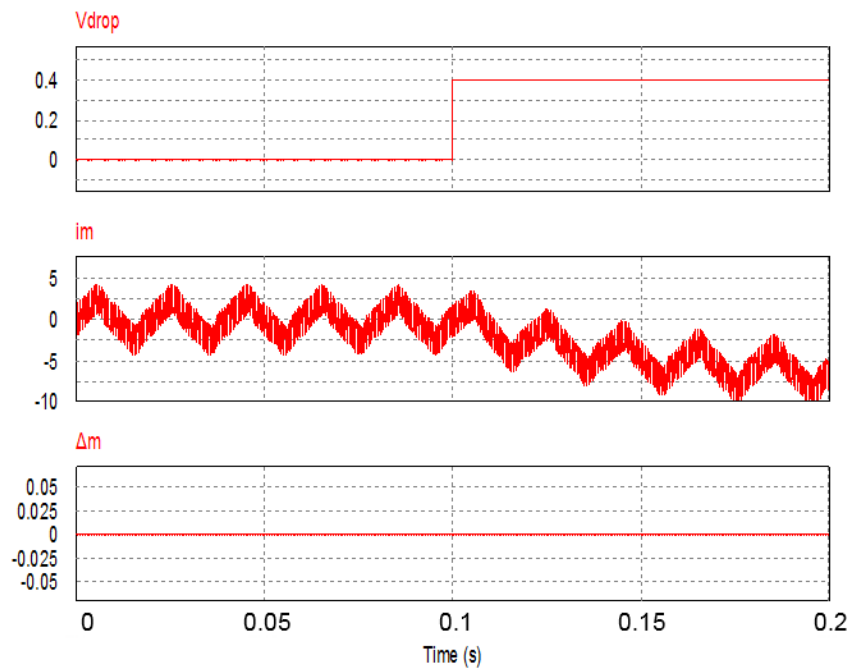

(a)
$C L_{R E S}(s)$ is shown in Fig. 7, where it can be seen that a high harmonic rejection is achieved.

In order to validate the proposed control technique, simulations are carried out, in which an additional dc-voltage drop, $V_{\text {drop }}$, is introduced after the H-bridge, in series with the HF transformer.

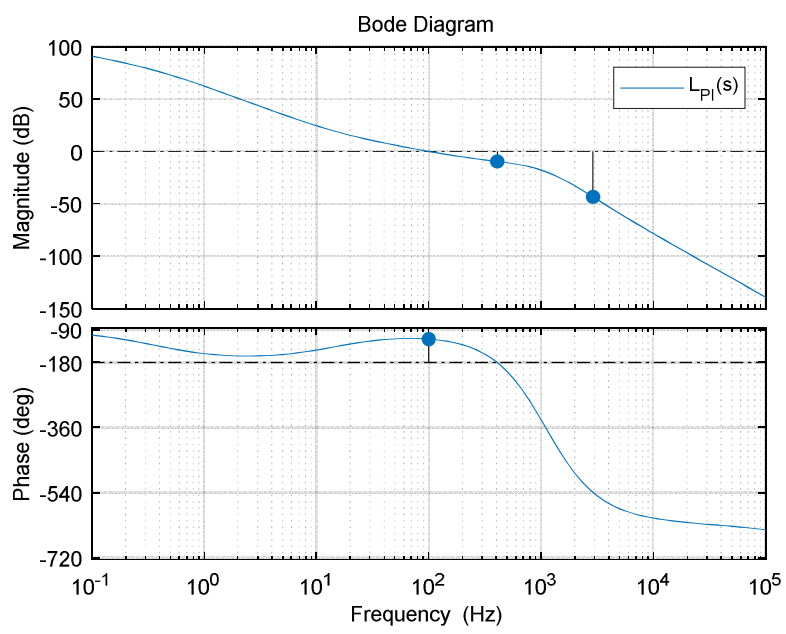

Fig. 6. Bode diagram of $\mathrm{L}_{\mathrm{PI}}(\mathrm{s})$ with the designed PI controller

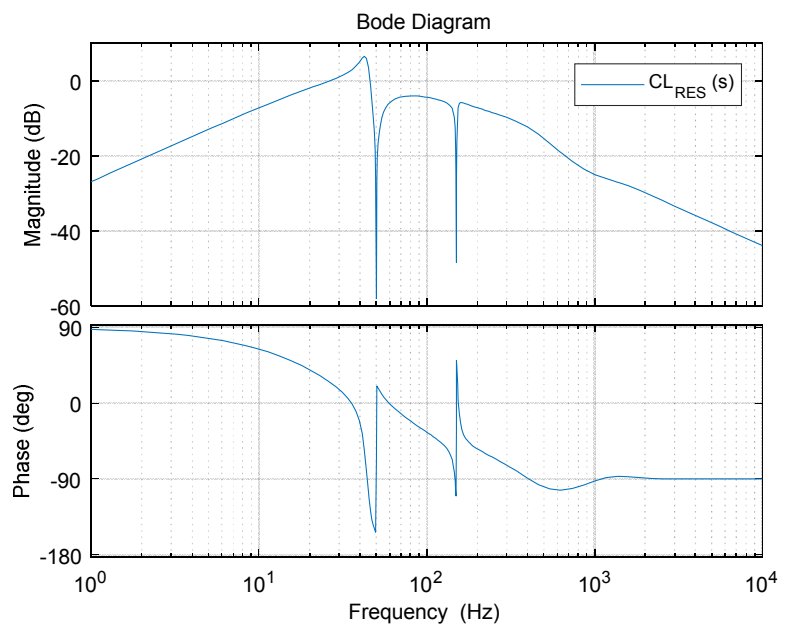

Fig. 7. Bode diagram of $\mathrm{CL}_{\mathrm{RES}}(\mathrm{s})$ with the designed resonant terms
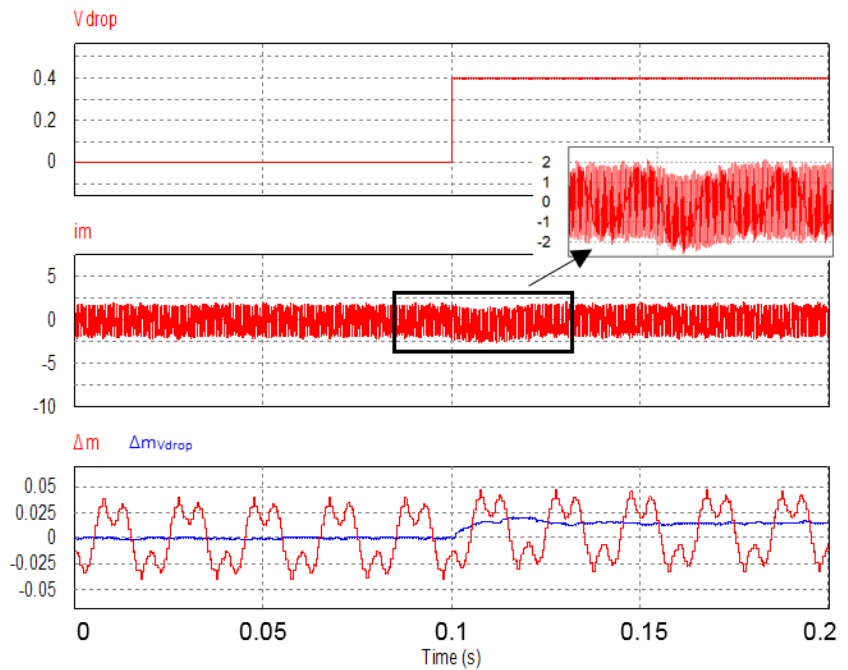

(b)

Fig. 8. Simulation results when: (a) the control technique is not enabled, (b) the control technique is enabled 
Fig. 8 (a) shows the simulation results of the additional dcvoltage drop, $V_{d r o p}$, the magnetizing current, $i_{m}$, and the modulation index variation, $\Delta m$, when the control technique to regulate the $\mathrm{dc}$ value of the magnetizing current is not enabled. Fig. 8 (b) shows the results when the proposed control technique is enabled.

Without enabling the control loop and before introducing the additional voltage drop, $i_{m}$ is centered with respect to zero, but it has low-frequency components at $50 \mathrm{~Hz}$ and at $150 \mathrm{~Hz}$, caused by the modulation technique. When the voltage drop is introduced, the magnetizing current decreases very fast, what will cause the transformer saturation.

By contrast, when the proposed control technique is enabled, $i_{m}$ is always centered with respect to zero. Before introducing the additional voltage drop, the control loop compensates the low-frequency harmonics thanks to the resonant terms, obtaining a magnetizing current with no lowfrequency components. When the additional voltage drop is introduced, it is also compensated by the control loop, maintaining the magnetizing current centered with respect to zero. A blue line, $\Delta m_{V \text { drop }}$, is depicted in Fig. 8 (b) to see only the effect of $V_{\text {drop }}$ in $\Delta m$.

The simulation results validate the proposed control technique. Besides, the efficiency of the converter remains almost unaltered, since less than $1 \mathrm{~W}$ is dissipated in the shunt resistor (i.e. less than the $0.33 \%$ of the prototype rated power). In addition, with the proposed technique the transformer does not have to be modified and only simple auxiliary circuits are needed to measure the magnetizing current.

\section{CONCLUSION}

In this paper, the flux-balancing of medium frequency transformers in a novel three-phase topology for cascaded conversion structures is studied. Based on the modulation of the converter, a technique that measures the magnetizing current three times per switching period is proposed. This technique improves previous saturation-detection methods, as it does not compromise the power-density of the converter and only requires simple auxiliary circuits. Moreover, a control loop to regulate the dc component of the magnetizing current is also developed and designed. Simulation results demonstrate that it can effectively compensate volts-seconds imbalances, preventing the transformer saturation.

\section{ACKNOWLEDGMENT}

The authors acknowledge the financial and continuous support of INGETEAM POWER TECHNOLOGY.

\section{REFERENCES}

[1] REN 21, "Ren21: Renewables 2019 global status report," 2019. [Online]. Available: https://www.ren21.net/gsr-2019/.

[2] R. M. Burkart and J. W. Kolar, "Comparative $\eta-\rho-\sigma$ Pareto Optimization of Si and SiC Multilevel Dual-Active-Bridge Topologies With Wide Input Voltage Range," IEEE Trans. Power Electron., vol. 32, no. 7, pp. 5258-5270, 2017.

[3] E. L. Barrios, A. Urtasun, A. Ursúa, L. Marroyo, and P. Sanchis, "HighFrequency Power Transformers With Foil Windings: Maximum Interleaving and Optimal Design," IEEE Trans. Power Electron., vol. 30 , no. 10 , pp. 5712-5723, Oct. 2015.

[4] M. Rabiul Islam, A. M. Mahfuz-Ur-Rahman, K. M. Muttaqi, and D. Sutanto, "State-of-the-Art of the Medium-Voltage Power Converter Technologies for Grid Integration of Solar Photovoltaic Power Plants," IEEE Trans. Energy Convers., vol. 34, no. 1, pp. 372-384, 2019.

[5] G. Ortiz, L. Fässler, J. W. Kolar, and O. Apeldoorn, "Flux Balancing of Isolation Transformers and Application of 'The Magnetic Ear' for Closed-Loop Volt-Second Compensation," IEEE Trans. Power Electron., vol. 29, no. 8, pp. 4078-4090, 2014.

[6] D. Lumbreras, E. L. Barrios, J. Balda, R. Gonzalez, and P. Sanchis, "Novel three-phase topology for cascaded multilevel medium-voltage conversion systems in large-scale PV plants," in 2020 IEEE 21th Workshop on Control and Modeling for Power Electronics (COMPEL), 2020, (in press).

[7] Seung-Gi Jeong and Min-Ho Park, "The analysis and compensation of dead-time effects in PWM inverters," IEEE Trans. Ind. Electron., vol. 38, no. 2, pp. 108-114, 1991.

[8] S. Klopper and J. A. Ferreira, "A sensor for balancing flux in converters with a high-frequency transformer link," IEEE Trans. Ind. Appl., vol. 33, no. 3, pp. 774-779, 1997.

[9] R. Patel, "Detecting impending core saturation in switched-mode power converters," in Proc. of the 7th National Solid-State Power Conversion Conference (POWERCON), 1980, vol. 3, pp. 1-11.

[10]D. Wilson, "A New Pulsewidth Modulation Method Inherently Maintains Output Transformer Flux Balance," Proc. 8th Natl. Solid-State Power Convers. Conf., vol. D1, no. April, pp. 1-14.

[11]J. L. Agorreta, M. Borrega, J. López, and L. Marroyo, "Modeling and Control of N-Paralleled Grid-Connected Inverters with LCL Filter Coupled Due to Grid Impedance in PV Plants," IEEE Trans. Power Electron., vol. 26, no. 3, pp. 770-785, 2011.

[12]A. G. Yepes, "Digital Resonant Current Controllers for Voltage Source Converters," Ph.D Thesis, p. 215. 